\title{
Technology-based cognitive training and rehabilitation interventions for individuals with mild cognitive impairment: a systematic review
}

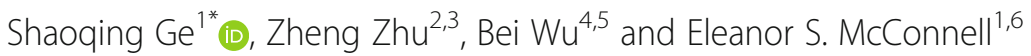

\begin{abstract}
Background: Individuals with mild cognitive impairment $(\mathrm{MCl})$ are at heightened risk of developing dementia. Rapid advances in computing technology have enabled researchers to conduct cognitive training and rehabilitation interventions with the assistance of technology. This systematic review aims to evaluate the effects of technologybased cognitive training or rehabilitation interventions to improve cognitive function among individuals with $\mathrm{MCl}$.

Methods: We conducted a systematic review using the following criteria: individuals with $\mathrm{MCl}$, empirical studies, and evaluated a technology-based cognitive training or rehabilitation intervention. Twenty-six articles met the criteria.

Results: Studies were characterized by considerable variation in study design, intervention content, and technologies applied. The major types of technologies applied included computerized software, tablets, gaming consoles, and virtual reality. Use of technology to adjust the difficulties of tasks based on participants' performance was an important feature. Technology-based cognitive training and rehabilitation interventions had significant effect on global cognitive function in 8 out of 22 studies; 8 out of 18 studies found positive effects on attention, 9 out of 16 studies on executive function, and 16 out of 19 studies on memory. Some cognitive interventions improved non-cognitive symptoms such as anxiety, depression, and ADLs.

Conclusion: Technology-based cognitive training and rehabilitation interventions show promise, but the findings were inconsistent due to the variations in study design. Future studies should consider using more consistent methodologies. Appropriate control groups should be designed to understand the additional benefits of cognitive training and rehabilitation delivered with the assistance of technology.
\end{abstract}

Keywords: Technology, Cognition, Cognitive training, Cognitive rehabilitation, Systematic review

\section{Background}

Due to the aging of the world's population, the number of people who live with dementia is projected to triple to 131 million by the year 2050 [1, 2]. Development of preventative strategies for individuals at higher risk of developing dementia is an international priority [3, 4]. Mild cognitive impairment (MCI) is regarded as an intermediate stage between normal cognition and dementia $[5,6]$. Individuals with MCI usually suffer with

\footnotetext{
* Correspondence: shaoqing.ge@duke.edu

'Duke University School of Nursing, 307 Trent Drive, Durham, NC, USA Full list of author information is available at the end of the article
}

significant cognitive complaints, yet do not exhibit the functional impairments required for a diagnosis of dementia. These people typically have a faster rate of progression to dementia than those without MCI [5], but the cognitive decline among MCI subjects has the potential of being improved $[7,8]$. Previous systematic reviews of cognitive intervention studies, both cognitive training and cognitive rehabilitation, have demonstrated promising effects on improving cognitive function among subjects with MCI $[3,7,9,10]$.

Recently, rapid advances in computing technology have enabled researchers to conduct cognitive training and rehabilitation interventions with the assistance of

(c) The Author(s). 2018 Open Access This article is distributed under the terms of the Creative Commons Attribution 4.0 International License (http://creativecommons.org/licenses/by/4.0/), which permits unrestricted use, distribution, and 
technology. A variety of technologies, including virtual reality (VR), interactive video gaming, and mobile technology, have been used to implement cognitive training and rehabilitation programs. Potential advantages to using technology-based interventions include enhanced accessibility and cost-effectiveness, providing a user experience that is immersive and comprehensive, as well as providing adaptive responses based on individual performance. Many computerized cognitive intervention programs are easily accessed through a computer or tablet, and the technology can objectively collect data during the intervention to provide real-time feedback to participants or therapists. Importantly, interventions delivered using technology have shown better effects compared to traditional cognitive training and rehabilitation programs in improving cognitive function and quality of life [11-13]. The reasons for this superiority are not well-understood but could be related to the usability and motivational factors related to the real-time interaction and feedback received from the training system [13].

Three recent reviews of cognitive training and rehabilitation for use with individuals with MCI and dementia suggest that technology holds promise to improve both cognitive and non-cognitive outcomes [14-16]. The reviews conducted by Coyle, et al. [15] and Chandler, et al. [14] were limited by accessing articles from only two databases, and did not comprehensively cover available technologies. Hill, et al. [16] limited their review to papers published until July 2016 and included only older adults aged 60 and above. More technology-based intervention studies have been conducted since then, and only including studies with older adults 60 and above could limit the scope of the review given that adults can develop early-onset MCI in their 40s [17]. Therefore, the purpose of this review is to 1) capture more studies using technology-based cognitive interventions by conducting a more comprehensive search using additional databases 2) understand the effect of technology-based cognitive interventions on improving abilities among individuals with $\mathrm{MCI}$; and 3) examine the effects of multimodal technology-based interventions and their potential superiority compared to single component interventions.

\section{Methods}

\section{Search strategy}

PRISMA guidelines were followed for conducting this systematic review [18]. Based on the research aims and key words, an experienced librarian searched five databases: PubMed (Medline), PsychoINFO (EBSCOhost), CINAHL (EBSCOhost), Embase, and Cochrane Library (Wiley). The search strategy used a combination of subject headings and key words for these main concepts: technology, MCI, training, and rehabilitation. The full search strategy is available in Additional file 1. The literature search was limited to empirical studies among human subjects. We did not set boundaries on age since MCI can occur among middle aged to older adults. The literature search was completed on December 1, 2017.

\section{Inclusion and exclusion of publications}

Two authors (SG and ESM) independently reviewed the list of articles found in the literature search. Inclusion criteria were: 1) participants were diagnosed with $\mathrm{MCI}$; 2) a technology-based cognitive training or rehabilitation intervention was evaluated; and 3) an empirical study was conducted. Exclusion criteria were: 1) the effect of the intervention on MCI participants could not be extracted from effects among healthy or dementia participants, and 2) the publication was not in English.

Titles were first reviewed for obvious exclusions. Then, for those retained from the first-round title screening, abstracts were screened. A third-round of full-text screening was then conducted. Any uncertainties or discrepancies between the two authors (SG and ESM) were discussed and resolved.

\section{Quality assessment}

The quality of studies identified as relevant was assessed by two independent reviewers (SG and $\mathrm{ZZ}$ ) using the Joanna Briggs Institute (JBI) critical appraisal checklist for randomized controlled trials (RCT) and JBI checklist for quasi-experimental studies [19]. Any disagreements that arose were resolved through discussion, or with a third reviewer (BW). The studies were generally methodologically sound with some variations in quality across studies (see Additional file 1: Table S2 and S3).

\section{Data extraction and synthesis}

Two reviewers (SG and ZZ) independently extracted information from each article into the Tables 1 and 2 . Disagreements on data extraction were resolved by consensus with the assistance of a third author. A meta-analysis of the 26 articles was inappropriate due to the large variability between the study designs, intervention contents, outcomes measured, and population samples across different studies [20,21]. We selectively calculated effect sizes for a pair of studies that used the same intervention materials [22, 23]. All data syntheses were conducted by using Revman 5.3 [24]. The forest plot is presented in Additional file 1: Figure S1.

\section{Results}

Based on the strategy and criteria described above, 26 of 411 studies identified were deemed eligible for review. The PRISMA flowchart in Fig. 1 presents the decision pathway for final inclusion of studies. 


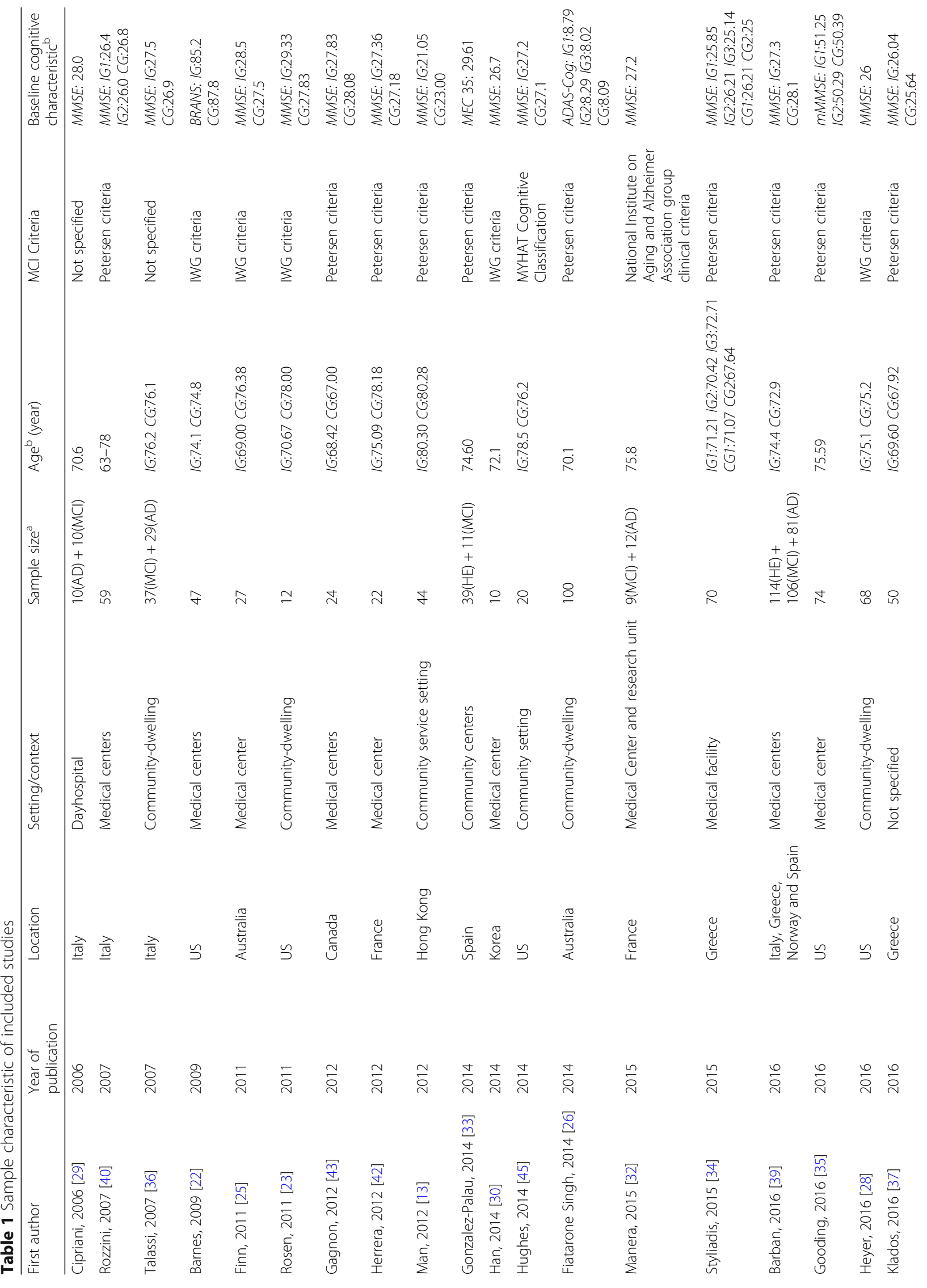




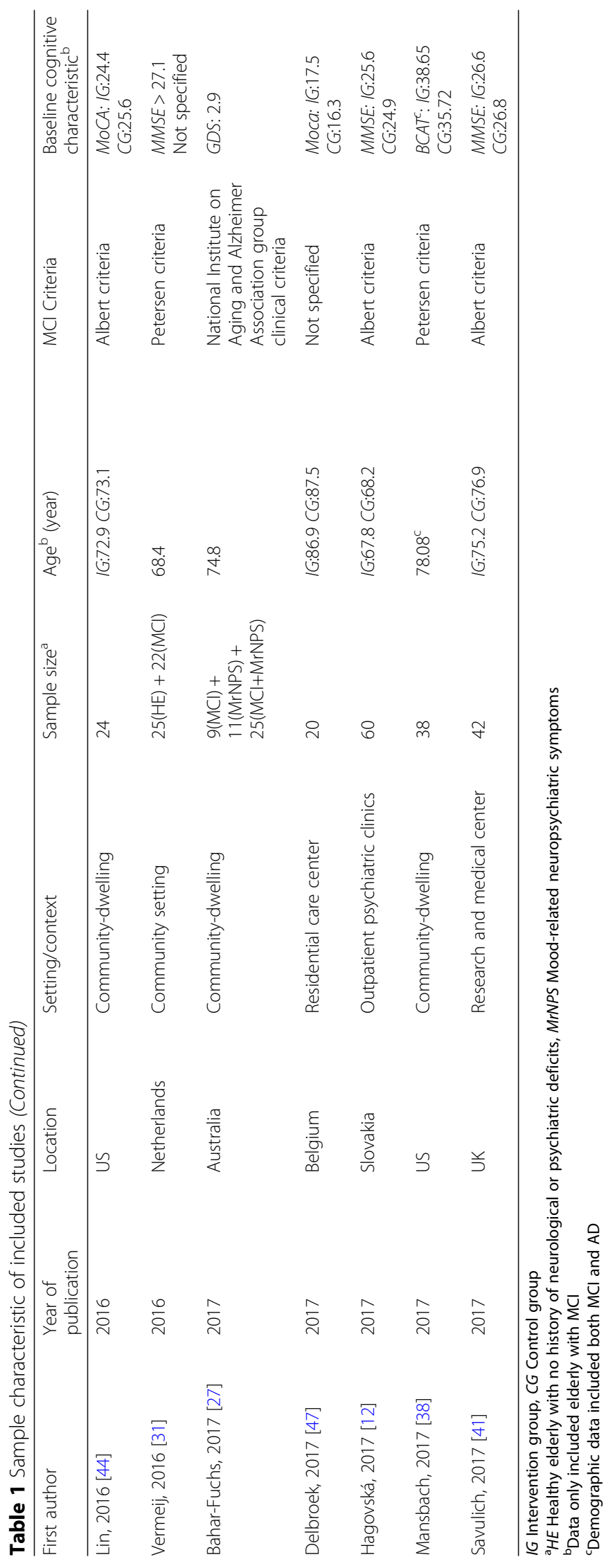




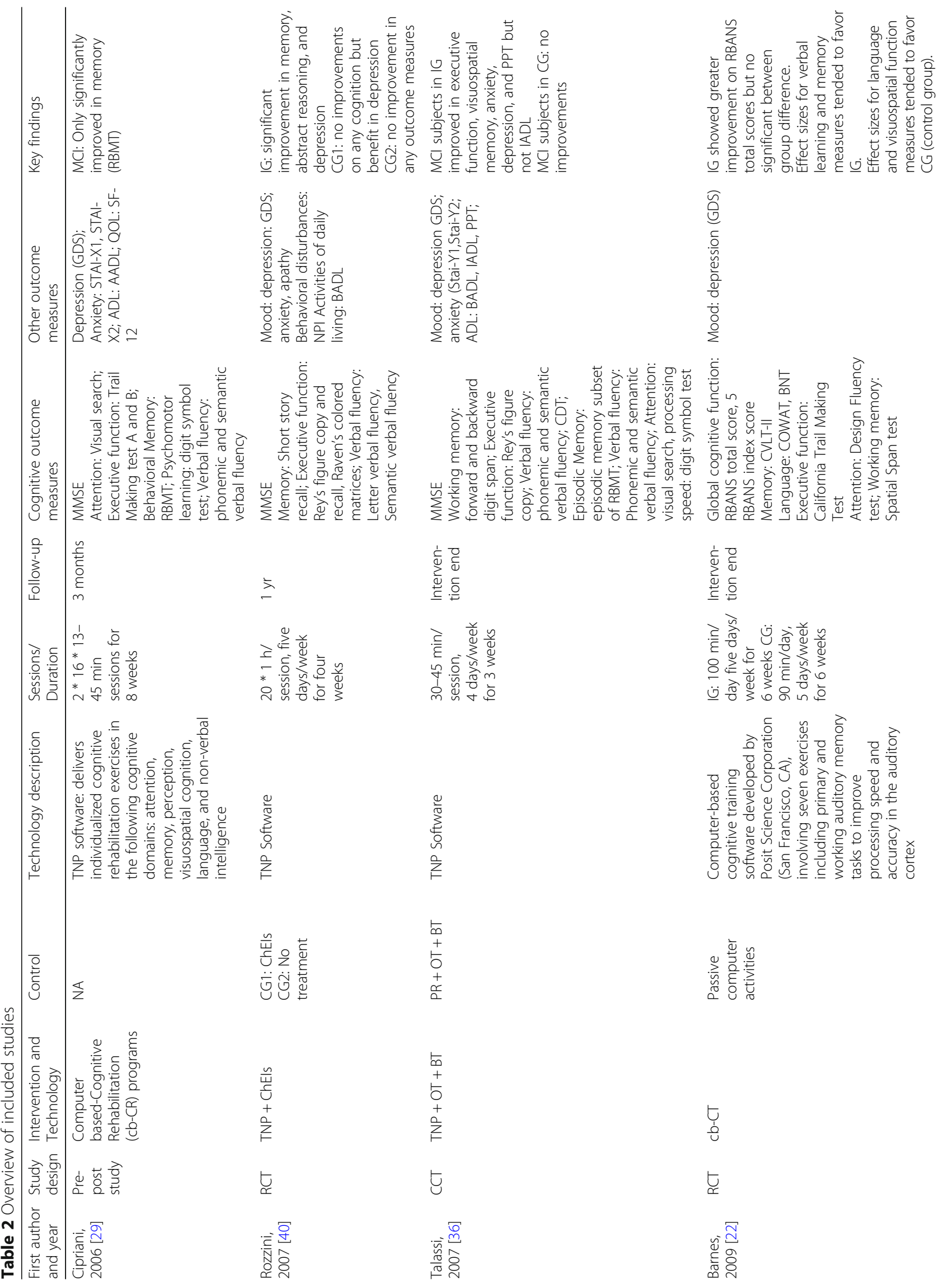




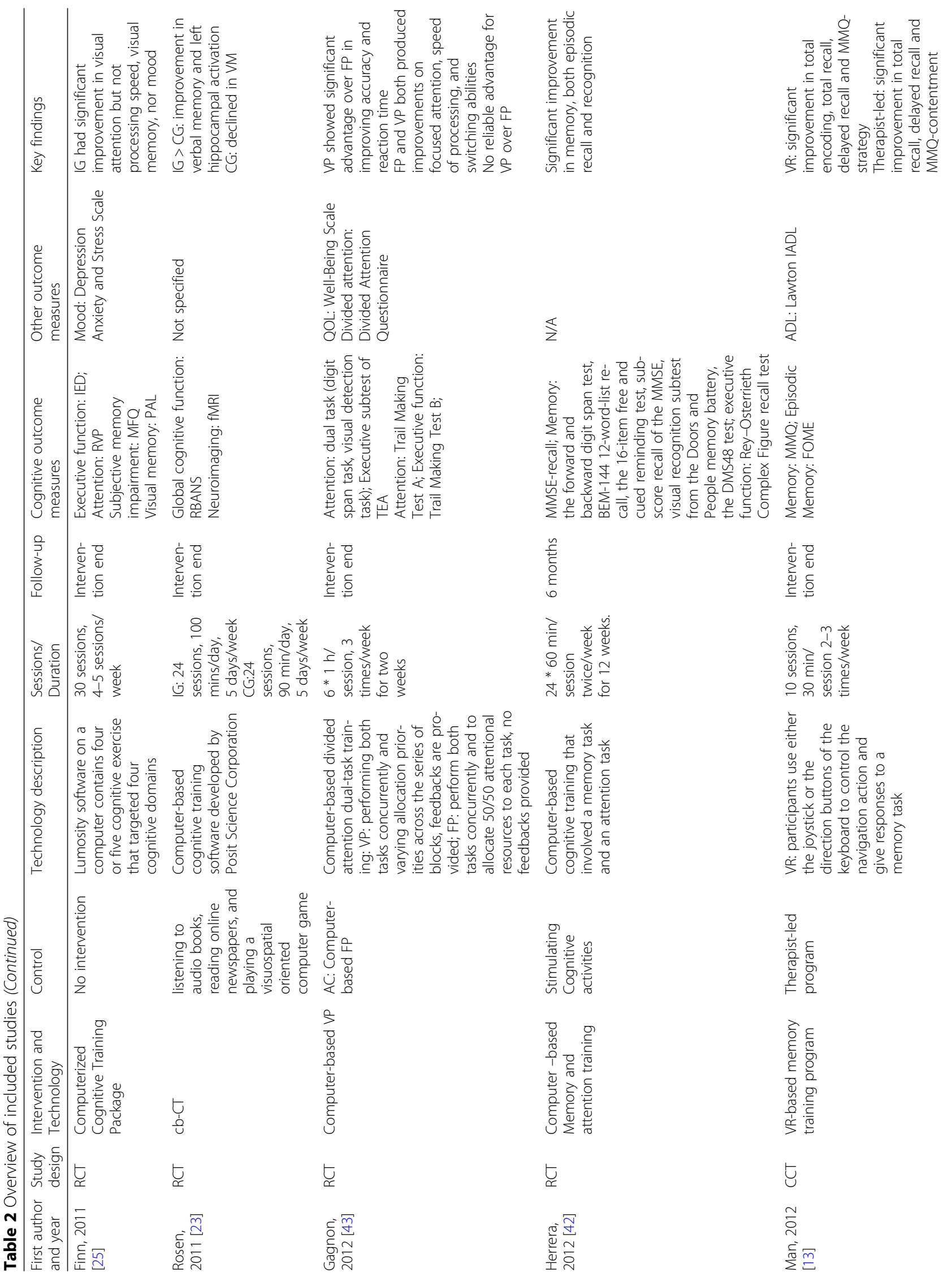




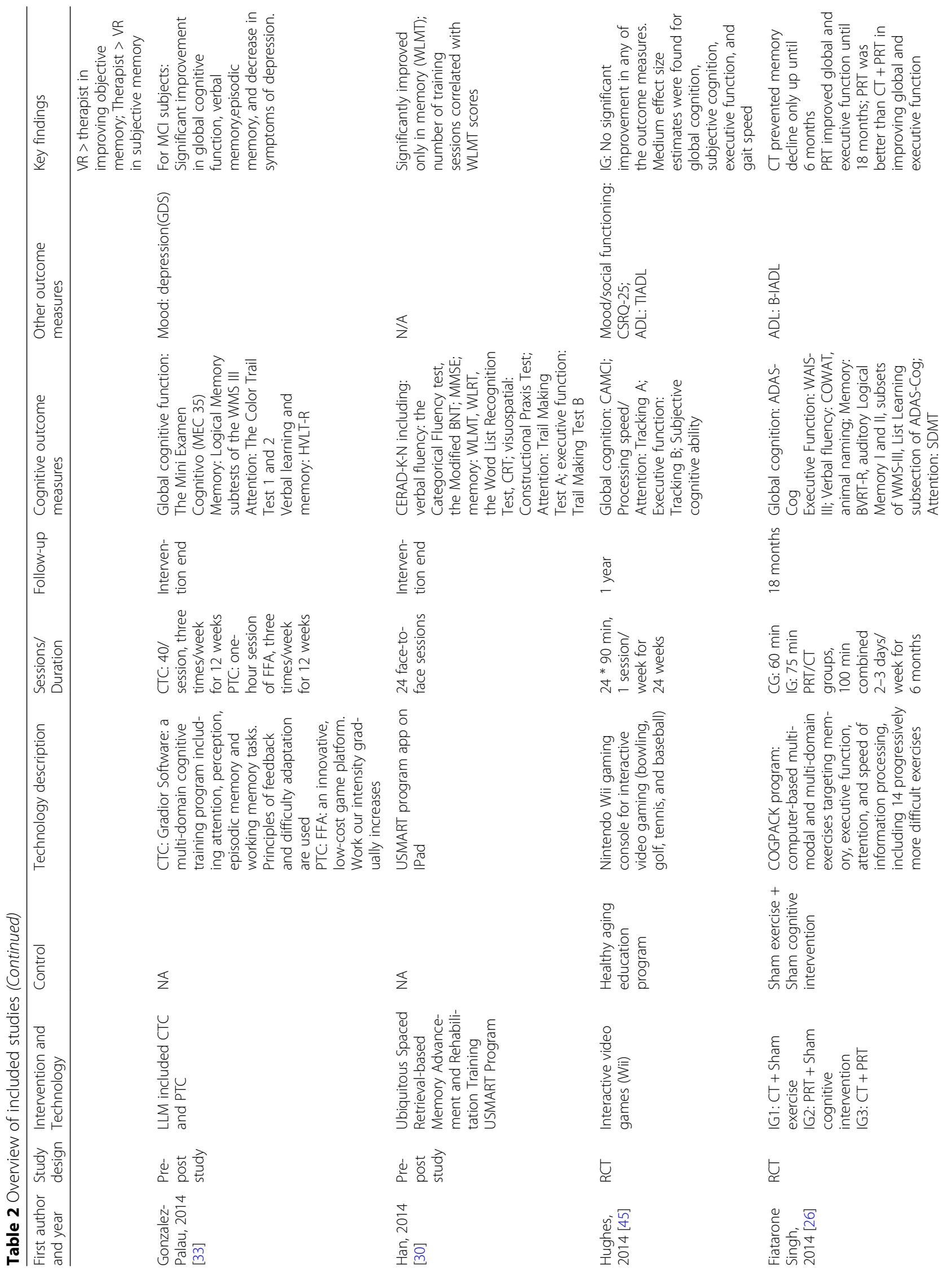




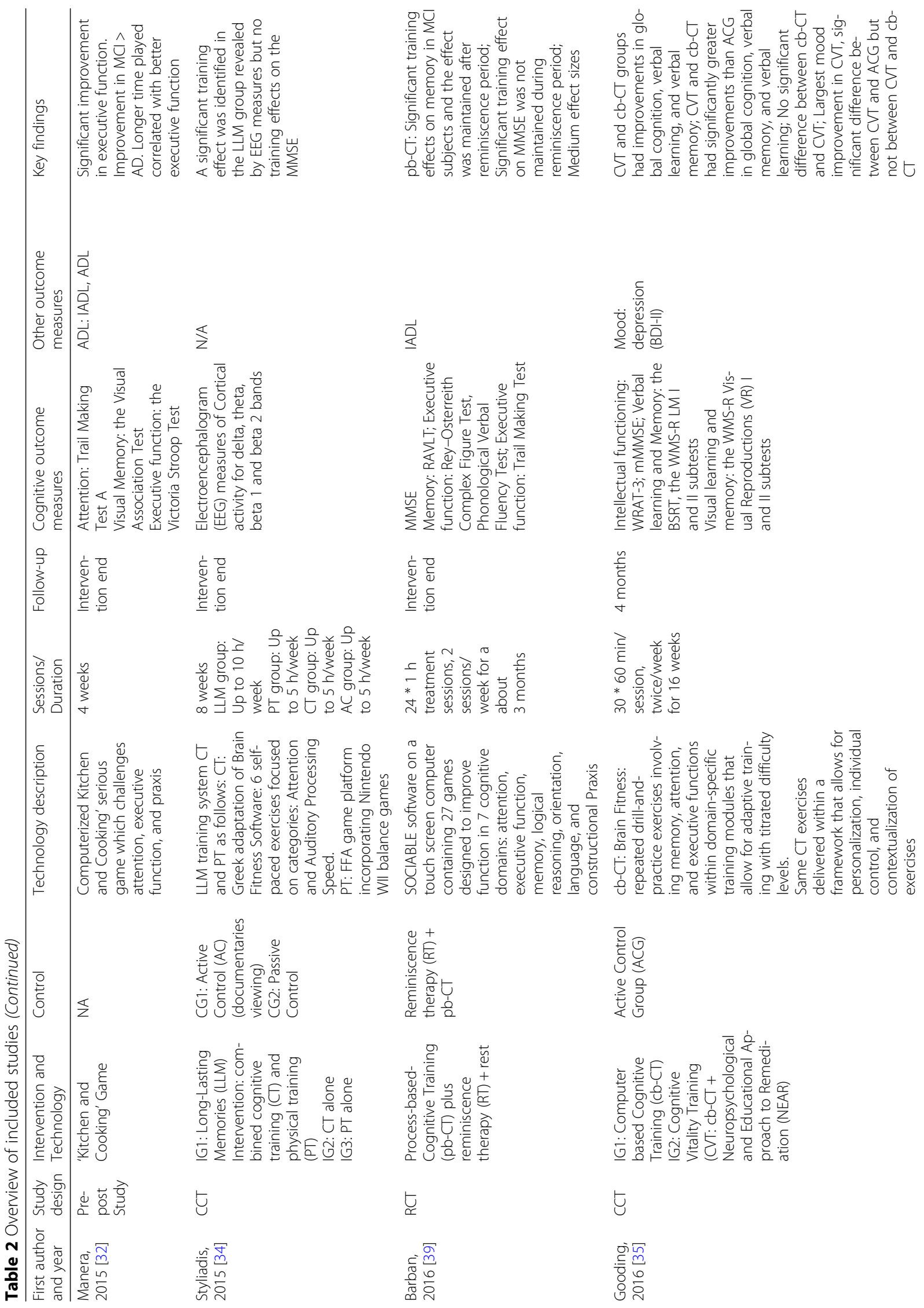




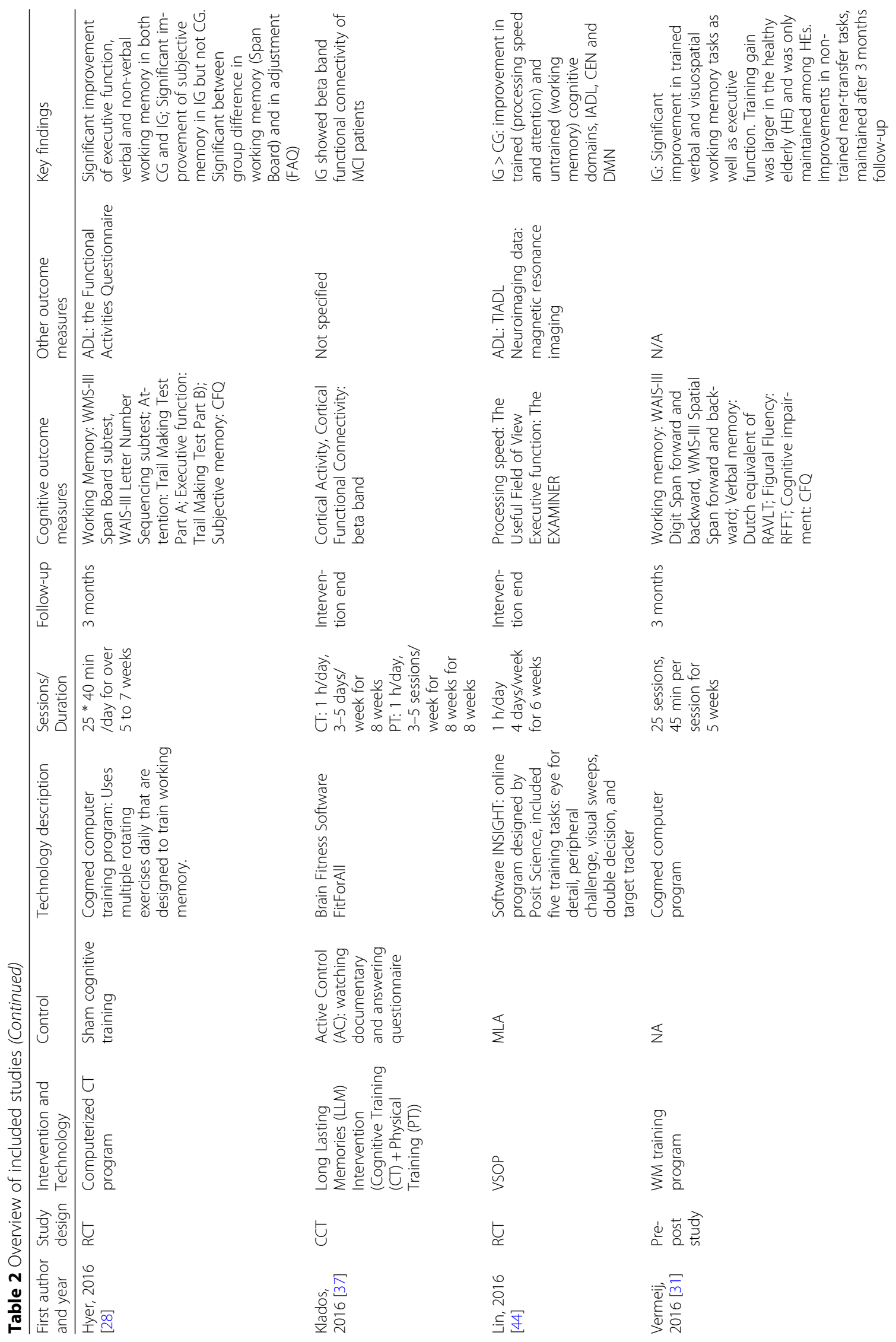




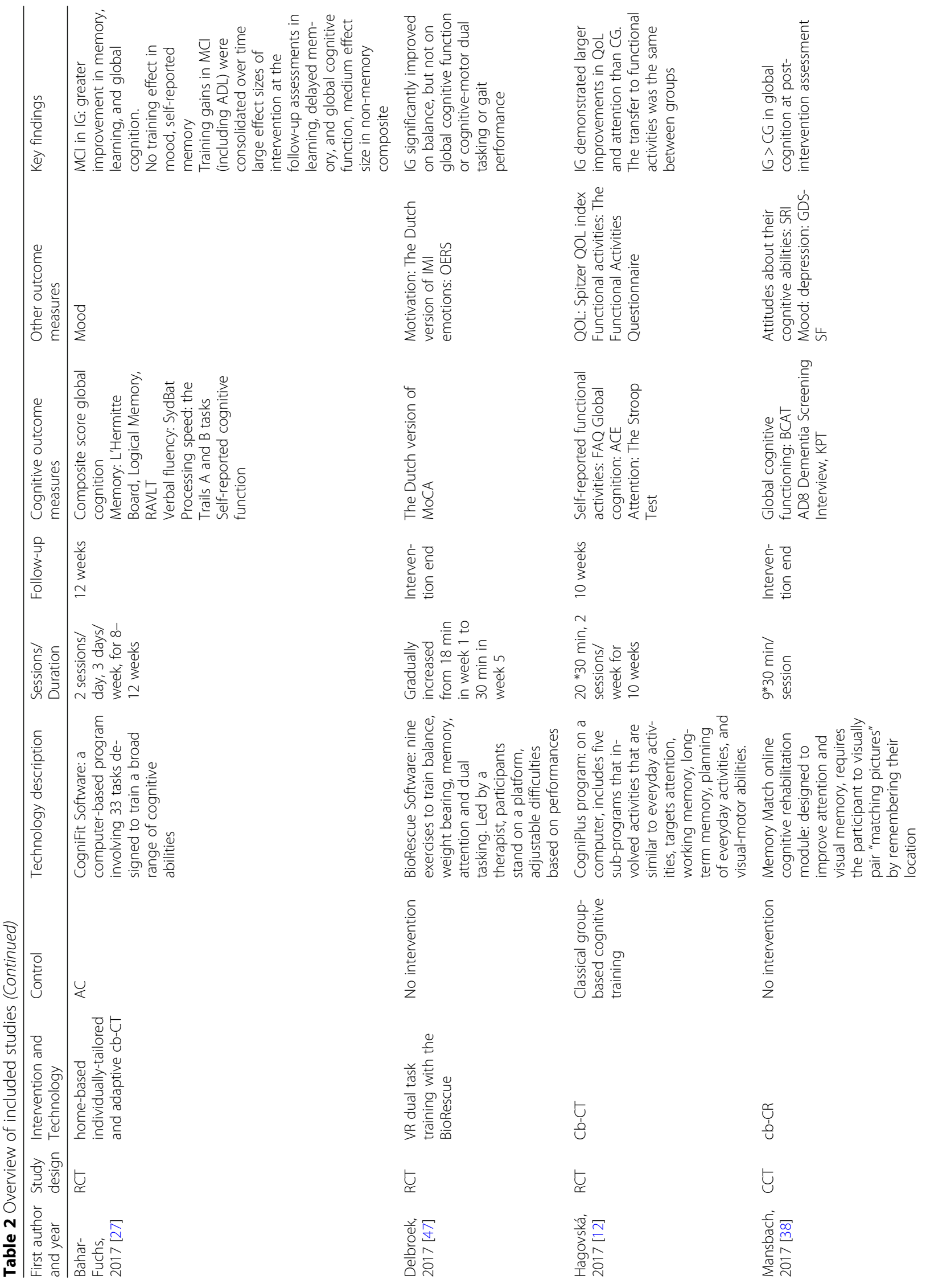




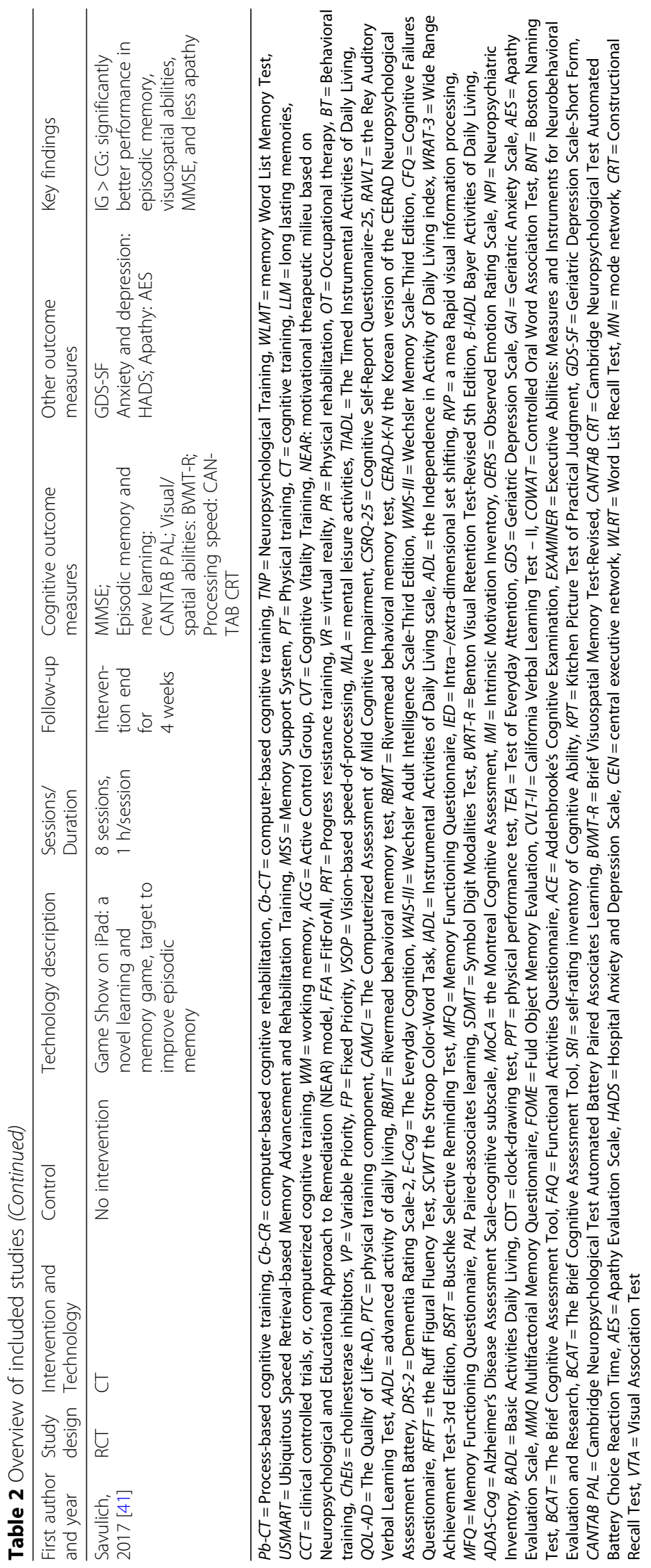




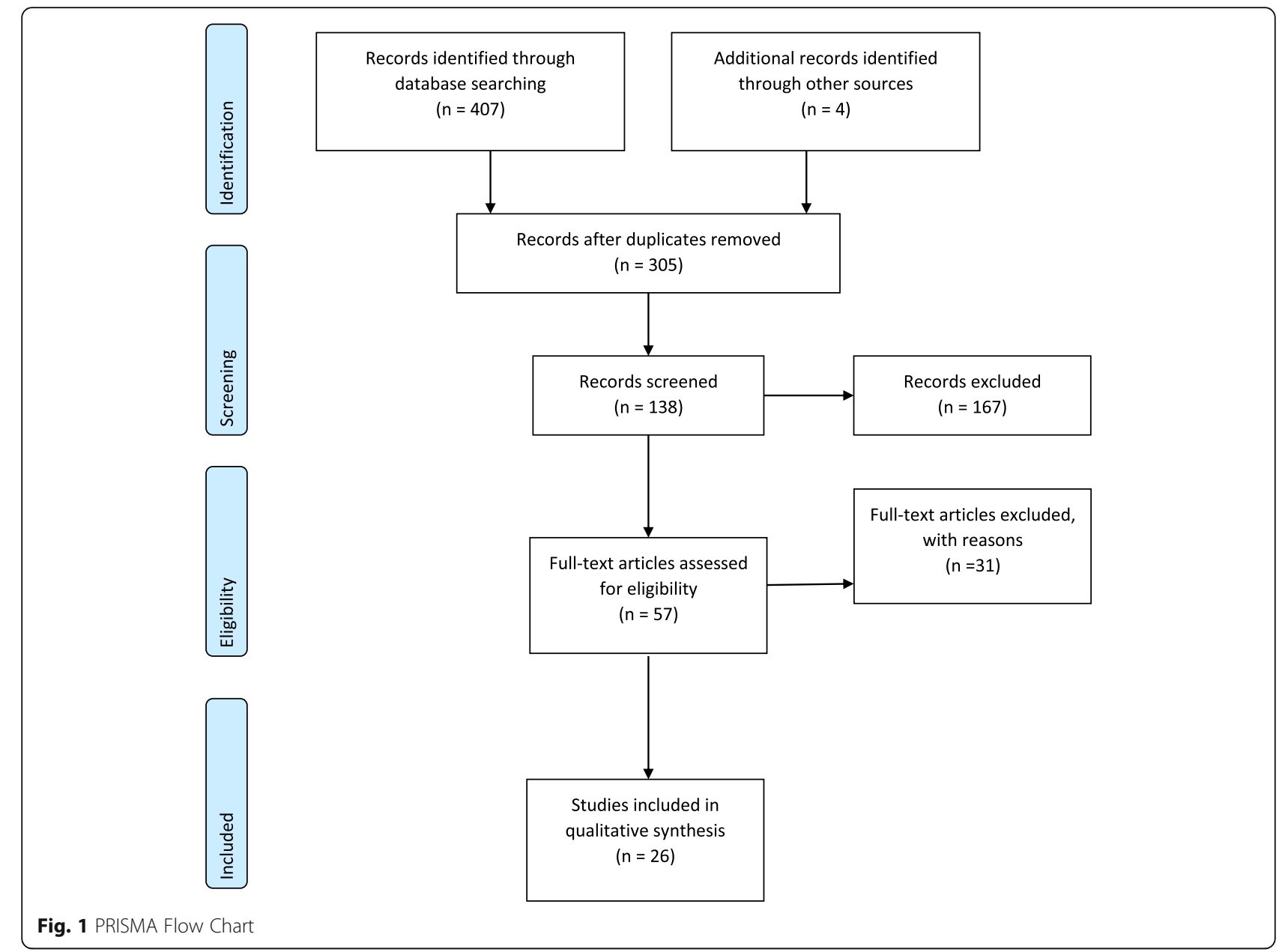

\section{Quality of the studies}

Additional file 1: Tables S2 and S3 summarize the quality assessment of the 26 eligible studies using the JBI criteria, which included both randomized controlled trials and quasi-experimental studies. Of these, 15 were randomized controlled trials (Table 2), 6 were controlled clinical trials, 5 were pretest-posttest studies (Table 2).

Among the RCT studies, only four articles [12, 25-27] reported the procedure for randomization. Two studies allocated the participants by utilizing computer-generated random numbers $[26,27]$, while the other two studies $[12,25]$ randomized participants by having an independent person use sealed envelopes. The remaining 11 articles did not report the randomization procedure used for allocating participants. Only three studies [26-28] reported that they were double-blinded sham-control trials.

In the quasi-experimental studies, five studies [29-33] utilized pretest-posttest design. The convenience samples and limited sample sizes $(n=10 ; n=10 ; n=22$; $n=9 ; n=11)$ restricted their generalizability. Six studies utilized a controlled clinical trial design [13, 34-38]. All the studies lacked external validity due to use of convenience samples or sampling methods that were not clearly described. Quasi-experimental studies which lacked randomization also were limited by a potential allocation bias.

\section{Sample characteristics of the included studies}

The sample and design characteristics of each study are summarized in Table 1 . More than $40 \%$ of the included studies were published from 2016 to $2017 \quad(n=11)$. Studies were conducted in different countries: United States $(n=7)$, Italy $(n=3)$, Australia $(n=3)$, France $(n=2)$, Greece $(n=2)$, Canada $(n=1)$, Hong Kong $(n=1)$, United Kingdom $(n=1)$, Belgium $(n=1)$, Slavonia $(n=1)$, Spain $(n=1)$, South Korea $(n=1)$, and Netherlands $(n=1)$. Only one study [39] reported recruitment across multiple countries.

The total number of the participants with MCI included in this systematic review was 1040. Seven studies included both participants living with MCI and those with other cognitive statuses (either individuals who were cognitively normal or individuals who had dementia), and reported intervention effects for groups of MCI individuals. The 
mean age ranged from 67.8 and 87.5. All but three studies reported the criteria used to diagnose MCI. Peterson criteria were used in 12 studies, International Working Group (IWG) criteria were used in five studies, Albert criteria were used in three studies, National Institute on Aging and Alzheimer Association workgroup clinical criteria were used in two studies, and MonongahelaYoughiogheny Healthy Aging Team (MYHAT) Cognitive Classification criteria were used in one study.

\section{Characteristics of interventions \\ Single-component interventions}

The majority of the studies ( $n=18$, or $69 \%$ ) involved single-component technology-based cognitive interventions (Table 2). Characteristics of the interventions varied widely. Seventeen different interventions were utilized in the cognitive training programs studied (see details in Table 2).

\section{Multimodal interventions}

Eight studies utilized multimodal interventions (Table 2). One approach was cognitive training combined with different types of therapies [35, 36, 39, 40]; another approach was cognitive training combined with physical training [26, 33, 34, 37].

Cognitive training plus therapy Four studies combined the technology-based cognitive training with other therapies as intervention, specifically reminiscence therapy [39], Neuropsychological and Educational Approach to Remediation (NEAR) [35], occupational therapy [36], and medications (cholinesterase inhibitors (ChEIs)) [40]. Two types of software were involved in the cognitive intervention component, including SOCIABLE [39], and NeuroPsychological Training (TNP) [36, 40]. The training sessions lasted for a minimum of 3 weeks [36] to a maximum of 16 weeks [35].

\section{Cognitive training combined with physical training} Four studies examined the combined effects of technology-based cognitive and physical training. Two studies used the Long-Lasting Memories (LLM) intervention to provide integrated cognitive and physical training [34, 37], and the physical component was delivered using the FitForAll platform. Gonzalez-Palau, et al. [33] also used the FitForAll platform to provide physical training, but used Gradior program to deliver the cognitive training. Singh and colleagues used Pneumatic resistance machines (Keiser Sports Health Equipment, Ltd) to provide progressive resistance training [26]. The length of these physical trainings lasted from 6 weeks [34] to 6 months [26].

\section{Overview of technologies}

The studies reviewed used the following types of technologies: traditional keyboard computers $(n=16)$, touch screen computers $(n=4)$, gaming consoles or platforms $(n=5)$, and tablets $(n=3)$. Gonzalez-Palau, et al. [33] and Styliadis [34] used both computer and gaming platforms in their interventions. Since 2014, technologies that are more interactive and immersive (virtual reality, gaming console, exergaming platform) have been introduced in cognitive intervention studies.

Compared to traditional therapist-led or pen and paper cognitive interventions, technologies are "smarter" in tracking participants' performances and adjusting the intervention difficulty. By applying technologies as a delivery method, researchers were able to record the participants' performance throughout the intervention process. Thirteen studies tracked participants' performance as one of the outcome variables. Twelve studies used intervention programs that could adjust the intervention difficulties to keep challenging the participants' abilities, as well as avoid distressing them with too many training failures.

\section{Effects of interventions \\ Cognitive outcomes}

Global cognitive function Twenty-two studies assessed the effects of the interventions on global cognitive function (Table 2). Various instruments were used, including MMSE, Repeatable Battery for Assessment of Neuropsychological Status (RBANS), Computerized Assessment of Mild Cognitive Impairment (CAMCI), Alzheimer's Disease Assessment scale-cognitive subscale (ADAS-Cog), Brief Cognitive Assessment Tool (BCAT), Addenbrooke's Cognitive Examination (ACE), Montreal Cognitive Assessment (MoCA), Spanish version MMSE (MEC35), and composite score from measured cognitive domains.

Out of the 22 studies, eight studies found their intervention significantly improved global cognitive function among individuals with MCI. These studies used different cognitive interventions, all but one [20] of them were interventions targeting multiple cognitive domains. Five studies used an active control group, and three of them found a significant between-group difference in global cognition improvement. Barban, et al. [39] reported a significant treatment effect of a computerized multi-domain process-based cognitive training combined with reminiscence therapy in MMSE mean scores (Cohen's $d=0.44$ ). Gonzalez-Palau, et al. [33] reported a significant improvement in global cognitive function (MEC35) among MCI individuals who went through a multi-domain cognitive training program including both cognitive and physical training components. Gooding, et al. [35] compared the 
computerized cognitive training and cognitive vitality training to an active control group, and reported significantly larger improvements in both intervention groups than the active control group in MMMSE mean score $\left[F(2,71)=11.56, p<0.001, \eta_{p}^{2}=0.25\right]$ with a medium effect size (Cohen's $d=0.30-0.53$ ). However, this training effect was not maintained at 3-month follow-up. Bahar-Fuchs, et al. [27] reported a significantly greater improvement in global cognition composite score in the training group than the active control with a large effect size (Cohen's $d=0.80$ ). On the other hand, Barnes, et al. [22] found significant RBANS total score improvement in the intervention group after an auditory processing speed and accuracy training, but the between-group difference compared to the active controls was not significant $(S D=0.33)$. All the other three studies that did not use an active control found significant between-group differences in changes in global cognitive function [26, 38, 41].

Two studies that compared the computer-based cognitive training with listening to audio books, reading online newspapers, and playing a visuospatially-oriented computer game met the requirement for meta-analysis $[22,23]$. The design of study, content of intervention, duration and length of follow-up were similar. A total of 59 individuals were included in the meta-analysis. In Additional file 1: Figure S1, the pool weighted standard mean difference score of RBANS total score was 1.62 (95\% CI: -1.63 - 4.87). This result indicated that there was no significant difference in the effectiveness for computer-based cognitive training in improving RBANS total score for individuals with MCI after intervention.

Attention and working memory Eighteen studies assessed the effects of technology-based cognitive training or rehabilitative programs on attention/working memory, which are required for storage of new information. The most commonly used measures were the digit span test. Other measures included the spatial span test, Trail Making Test A and B, visual search, spanboard, dual task (digit span task + visual detection task), subscale of Addenbrooke's Cognitive Examination, and Symbol Digit Modalities Test (SDMT).

Out of 18 studies, eight studies reported significant improvement in attention/working memory. Two studies compared computerized training programs (Cogmed Software) with no intervention or a sham cognitive intervention $[28,31]$. Significant improvements on spanboard $(p=.01)$ [28], digit span $(p<.01)$ [31], and spatial span $(p<.05)$ [31] performance were observed at a 3 -month follow-up in the intervention group. Other interventions included memory and attention training, variable priority training, and vision-based speed-of-processing training. Significant improvements were found in digit span forward ability $\left(\eta_{p}^{2}=.14, p<.05\right)$ [42], accuracy $(p=0.001)$, reaction time $(p<.01)$ [43], spatial span $(p=.003)$ [22] and working memory $\left(\eta_{p}^{2}=.28, p=.01\right)$ [44]. However, three other studies that measured attention using digit span did not report significant results $[23,33,36]$.

In terms of technologies used among the eight studies, all of them applied computerized programs to deliver the interventions. Specifically, they all used a keyboard, not a touch screen, to record the test responses.

Executive function Sixteen studies assessed the effect of technology-based cognitive intervention on executive function. Among them, six studies used the Trail Making Task B, six studies used the phonemic and semantic fluency test, four studies used the Rey-Osterreith Complex Figure Test. Other measures included: WAIS Matrices, Ruff Figural Fluency Test, Test of Everyday Attention (TEA), the intra-/extra-dimensional set shifting, and Executive Abilities: Measures and Instruments for Neurobehavioral Evaluation and Research (EXAMINER).

Out of the 16 studies, nine studies reported significant improvement in executive function [22, 26-28, 31, 32, $36,40,44]$. The interventions used in these studies included both multi-domain cognitive training, specific training tasks, and gaming. The length of interventions ranged from 3 [36] to 24 weeks [26]. Interestingly, three studies used TNP software as an intervention component [29, 36, 40]; although the intervention length varied, two out of the three studies found significant improvement in executive function but used different measures [36, 40]. Talassi [36] found that TNP integrated with occupational therapy and behavioral therapy had a significant improvement in the Rey-Osterreith Complex Figure Test. Rozzini and Costardi [40] found that MCI individuals receiving cognitive training and ChEIs reported significant improvements in Ravens Coloured Progressive Matrices post-intervention $(p<0.02)$. This beneficial effect was not found when using TNP only [29]. However, both Talassi [36] and Rozzini [40] failed to report an effect size for their intervention effect. Other studies that demonstrated significant improvements in executive function varied greatly in terms of sample size, intervention content, total intervention time, and executive function measures.

Memory Nineteen studies assessed memory. Sixteen out of the 19 studies found a significant effect on memory. The measures of memory varied greatly. Major measures included the Wechsler Memory Scale (WMS) and Rey Auditory Verbal Learning Test (RAVLT). Four studies 
used the WMS-III and three out of the four studies found significant improvements in memory after intervention. The intervention used in the three studies included Cogmed computer program [31], Game show on iPad [41], and LLM including both cognitive and physical training components [33]. The intervention period ranged from 5 to 12 weeks and significant improvement in memory was reserved until end of the three months' follow-up [31]. The other study used GOPACK multi-domain cognitive training program to conduct a 6-month intervention but did not find a significant improvement in memory measured by subsets of WMS-III [26]. Three studies used RAVLT to measure verbal memory and all of them found significant benefit of the cognitive interventions being used [27, 31, 39]. The interventions included SOCIABLE, Cogmed, and CogniFit software programs, with the intervention lengths ranged from 5 to 12 weeks and follow-up period up to 3 months [27, 31]. Among the three training software programs, Cogmed targeted on working memory, while the other two targeted on multiple cognitive domains.

Other studies that studied memory as an outcome variable each used various measures including the 12-word-list recall test from the BEM-144 memory battery, the 16-item free and cued reminding test, Buschke Selective Reminding Test, Hopkins Verbal Learning Test, Auditory Logical Memory, Short story recall, WMS-R Visual Reproductions I and II subtests, Pattern recognition memory, Benton Visual Retention Test, short story recall, Rivermead behavioral memory test, and Brief Visuospatial Memory Test-Revised. The interventions of these studies lasted for 3 [36] to 16 weeks [35] with the follow-up time up to 6 months. All but one [32] of these studies demonstrated significant improvements in memory. Manera et al. [32] found the 4-week "kitchen and cooking game" intervention had no significant effect on improving memory.

In terms of technologies, all but two studies used computer to deliver the interventions and used keyboard to collect the data. Only two studies used iPad [41] and VR technology [38] to deliver their memory training programs.

\section{Non-cognitive outcomes}

Mood Nine studies assessed depression. The most commonly used measures were Geriatric Depression Scale (GDS) and its short form GDS-SF [22, 29, 33, $36,38,40,41]$, other inventory used included Beck's Depression Inventory [35] and Depression Anxiety and Stress Scale [25]. Four studies reported significant reduction of depression among individuals with $\mathrm{MCI}$ $[33,35,36,40]$. None of these studies reported effect sizes for their interventions reducing depression. Two out of these four studies used a multimodal intervention that also integrated physical trainings $[33,36]$.

Five studies assessed anxiety [25, 29, 36, 40, 41]. The most often used measure was the State Trait Anxiety Inventory (STAI) $[29,36]$ used in two studies. Only one study showed a significant reduction in anxiety for individuals with MCI. Talassi used a multimodal intervention including cognition, behavioral, and occupational training compared to its control group that had physical rehabilitation, occupational, and behavioral training, and found that the intervention group had significant decrease in anxiety but not the control group participants [36].

ADL Eleven studies assessed ADL as a secondary outcome. The Basic Advanced and Instrumental Activities of Daily Living scales were used in 5 studies [29, 36, 40, $44,45]$. Other measures included the Functional Activities Questionnaire [28], B-IADL scale [26], and HK Lawton IADL [13]. Two out of nine studies reported a statistically significant improvement in ADL [27, 44]. However, only one study found a significant between-group difference with a small to medium effect size $\left(\eta^{2}=0.21\right)[44]$.

Quality of life Three studies assessed quality of life [12, 29, 43]. Measures included SF-12 [29], Well-Being Scale [43] and Spitzer-QOL [12]. Only one study reported significant results. Hagovská, et al. [12] found that technology-based cognitive training produced a larger improvement in QoL than classical group-based cognitive training with a medium effect size $(r=0.69)$.

\section{Discussion}

In the past decade, technology-based cognitive interventions have gained increased research interest worldwide. Almost half (42\%) of the studies reviewed were published in 2016 and 2017, suggesting the growth in the importance of technology-based interventions. The vast majority of the studies were conducted in developed countries, which may be associated with the limited availability of and familiarity with technology among older adults in lower income countries.

The types of technology used varied across studies and included computers, tablets, VR, and gaming consoles. Computers were the most widely used technology with $77 \%$ using computers to assist delivery of cognitive interventions. The majority utilized commercially available software or programs, with only nine of the studies used training programs developed by the study researchers for the specific study purpose. Therapists or coaches were used to teach, assist, or even supervise the use of technologies along the intervention process. In nearly half of studies $(n=12)$ therapists provided instructions 
at the beginning of the intervention, or provided help throughout the intervention. All but two studies were conducted using only one type of technology, so no conclusions can be drawn regarding the effect of different types of technology on intervention results. Comparing effects of technology across studies was not possible due to the variability among interventions. With the rapid development of technologies, we can anticipate new types of technologies being utilized to assist cognitive training and rehabilitation interventions in the future.

Overall, technology-based cognitive training and rehabilitation have demonstrated promising beneficial effects on various domains of cognition with moderate to large effect sizes. Most studies (e.g., [28, 31, 44]) assessed participants on different cognitive domains that were not limited to the trained task, but also in other non-trained tasks and other cognitive domains, suggesting a transferable beneficial effect of cognitive training and rehabilitation. For example, Hyer, et al. [28] found that working memory training also improved executive function among trained MCI participants, and the impact was preserved until the end of the 3-month follow-up. This transferability is consistent with previous systematic reviews $[14,15]$. However, the training gain and transferability of the training gain varied by intervention (e.g., [22]) and delivery method (e.g., [13]). Therefore, future studies are still needed to understand which intervention would benefit various cognitive domains most efficiently.

Only one study included in this review examined whether applying technology as the delivery method would have a stronger effect on the intervention outcomes, in comparison to the use of a traditional manual delivery. Man, et al. [13] compared the training effect of a memory training program delivered with a non-immersive VR-based system versus with color-print images that matched the VR images. This study found that the VR group showed greater improvement in objective memory but the non-VR group reported greater contentment with memory performance, highlighting the potential importance of receiving verbal and emotional support from training therapists on improving participants' satisfaction with their memory performance. This study shows that depending on the outcomes that an intervention targets, technology-based and manual trainings may have their own strengths and weaknesses. No conclusions can be made whether one type of intervention is generally more effective than the other.

The effect of the same technology-based cognitive intervention seems to vary between groups with different level of cognitive decline. Some but still limited evidence suggested that participants without cognitive impairment seem to obtain a larger cognitive improvement from technology-based cognitive interventions than those with MCI. Vermeij, et al. [31] found that healthy participants had a larger gain in both trained working memory tasks and untrained executive function tasks than those with cognitive impairment. However, the findings are not conclusive. Barban, et al. [39] found that process-based cognitive training improved verbal memory among MCI participants and improved executive function among healthy participants. On the other hand, participants with MCI seem to gain a larger cognitive benefit than those with Alzheimer's disease (AD). Cipriani, et al. [29] fount that the TNP program significantly improved memory and global cognition among participants with $\mathrm{MCI}$, but only improved memory among those with AD. Similarly, Manera, et al. [32] found that the serious cooking game significantly improved executive function among participants with $\mathrm{MCI}$ but not those with AD.

Measures of physical function and mood were used in most studies, but unfortunately most of these were used to ensure non-biased randomization assignment at baseline rather than as outcome measures, so we have limited understanding of the effects of technology-based cognitive interventions on these outcomes. Nine studies evaluated mood (e.g., depression, anxiety) as an outcome, and eleven studies included ADL as an outcome variable. Among these, four studies found technology-based cognitive interventions had beneficial effect on mood and two studies found beneficial effect on functional activity. Technology-based cognitive training studies included in this review may have limited impact on mood and functional activity.

Two studies of technology-based cognitive training and one study of technology-based cognitive rehabilitation examined the effect of their interventions on quality of life [12, 29,43]. However, only one of the four studies reported significant result. Hagovská, et al. [12] found that technology-based cognitive training produced a larger improvement in QoL than classical group-based cognitive training. This lack of effect of cognitive intervention on QoL is consistent with previous systematic review on the efficacy of cognitive interventions on QoL [14]. However, each study used a different QoL instruments, and various research designs (e.g., types of interventions, lengths of follow-up, and types of control groups). Given the limited number of studies conducted, future studies using comparable designs are still needed to further understand the effectiveness of the intervention on quality of life.

A previous systematic review suggested that multimodal cognitive inventions were a promising research area [15]. In our review, we found eight studies that applied multimodal interventions combining technology-based cognitive training and physical exercise or other therapeutic methods. We expected to see the findings of these studies 
would provide support for speculation that multimodal cognitive interventions would produce a greater impact on improving cognitive function as well as mood and functional abilities. However, the eight reviewed articles provided insufficient evidence to support this conjecture. Five out of the eight studies were not designed to compare the efficacy of multimodal cognitive intervention compared to cognitive intervention alone [33, 34, 36, 37, 39], and it was difficult to draw any conclusions from the remaining three studies remained due to the great variability in the designs across studies. According to one study, customized technology-based cognitive training produced additional benefit, and technology-based cognitive intervention plus ChEIs was superior to ChEIs alone [35]. Interestingly, Fiatarone-Singh, et al. [26] found that progressive resistance training produced more improvement in executive function and global cognition than both cognitive training and multimodal intervention including cognitive training and progressive resistance training. Findings from this study suggest that physical exercise may particularly benefit executive function, but that implementing multimodal cognitive and physical interventions may be too challenging for people with MCI. Previous systematic review on the efficacy of combined cognitive and exercise intervention in older adults with and without cognitive impairment did not find sufficient evidence to confirm the beneficial effect among older adults with cognitive impairment [46]. Taken together, more studies are needed to understand the advantages of a multimodal cognitive intervention in individuals with MCI. Future studies should design appropriate control groups to understand the additional value produced by a multimodal cognitive intervention than a single model intervention. Additionally, researchers should also bear in mind the possibility that older adults with MCI may not be able to manage the cognitive challenge associated with multimodal interventions.

The studies reviewed generally had small sample sizes, ranged widely from 10 [13] to 301 participants [39]. The average sample size across studies was $54 ; 39 \%$ of the studies had sample sizes of less than 30 . The small sample sizes may be related to the complicated diagnostic criteria of $\mathrm{MCI}$, the ethical challenges of conducting intervention studies in older adults with $\mathrm{MCI}$, and the limited availability of some technology-based cognitive intervention programs. More importantly, potential beneficial effects of an intervention could be diminished due to a small sample size.

The measures applied varied greatly across studies, which created challenges in the comparison and generalizability of the study findings. Future studies should consider using measures that have been shown to have good validity and reliability as well as have been frequently used among the MCI population (e.g., CES-D, MMSE, QOL-AD, etc.). Neuroimaging techniques have emerged to be more widely used to obtain information on how technology-based cognitive interventions would affect neural connectivity [37], activation [23], and brain atrophy [31].

We conducted a comprehensive search of literature on the topic area using five major databases. However, this systematic review should be considered in light of its limitations. We only reviewed articles in English language. There may be other relevant studies that were published in other languages. We also need to be aware that technology is developing rapidly so that promising technology-based cognitive training and rehabilitation programs may exist that have not yet been published due to concerns about protecting participants.

\section{Conclusion}

The findings from this systematic review suggest that technology-based cognitive training and rehabilitation programs show promise for improving cognitive function, with some interventions showing moderate to large effect sizes. Computers, tablets, gaming consoles and platforms, and VR systems were the common types of technologies used. Both general and domain-specific cognitive training have led to improved cognition, primarily in memory, but with some evidence that executive function may also be positively affected. Studies that examined the impact of cognitive training on improving mood and functional abilities, have generated less convincing evidence. Multimodal intervention programs integrated technology-based cognitive intervention and other therapies have produced inconsistent findings on the superiority over only applying technology-based single model cognitive intervention. Overall, technology-based cognitive training and rehabilitation are promising intervention methods to improve cognitive function. Future studies should put effort to clarify whether the added benefits of implementing multimodal interventions exist, and carefully consider the potential extra burden caused to individuals with MCI. Additionally, future studies should aim to lessen the variabilities in intervention design and measures applied.

\section{Additional file}

Additional file 1: Table S1. Searching Strategy. Table S2. Results of quality assessment based on JBI critical appraisal checklist for randomized controlled trials*. Table S3. Results of quality assessment based on JBI critical appraisal checklist for quasi-experimental studies*. Figure S1. Forest plot - RBANS score (computer-based intervention VS. Control), (DOCX $34 \mathrm{~kb})$

\section{Abbreviations}

ACE: Addenbrooke's Cognitive Examination; AD: Alzheimer's disease; ADASCog: Alzheimer's Disease Assessment scale-cognitive subscale; BCAT: Brief 
Cognitive Assessment Tool; CAMCl: Computerized Assessment of Mild Cognitive Impairment; ChEls: cholinesterase inhibitors; GDS: Geriatric Depression Scale; GDS-SF: Geriatric Depression Scale short form; IWG: International Working Group; JBI: Joanna Briggs Institute; LLM: LongLasting Memories; MMSE: Mini-mental state examination; MoCA: Montreal Cognitive Assessment; MYHAT: Monongahela-Youghiogheny Healthy Aging Team; NEAR: Neuropsychological and Educational Approach to Remediation; RAVLT: Rey Auditory Verbal Learning Test; RBANS: Repeatable Battery for Assessment of Neuropsychological Status; RCT: randomized controlled trial; SDMT: Symbol Digit Modalities Test; STAI: State Trait Anxiety Inventory; TEA: Test of Everyday Attention; TNP: Neuropsychological Training; VR: virtual reality; WMS: Wechsler Memory Scale

\section{Availability of data and materials}

All data generated or analysed during this study are included in this published article [and its supplementary information files].

\section{Authors' contributions}

SG designed and conducted the study, abstracted data, interpreted the results, and played a major role in writing the manuscript. ZZ provided methodological support, helped abstracted data, appraised quality, and helped wrote a certain section of the manuscript. BW helped conceive the study and edited the manuscript. ESM helped conceive the study, screened citations and full-text articles, and edited the manuscript. All authors read and approved the final manuscript.

\section{Ethics approval and consent to participate}

Not applicable.

\section{Consent for publication}

Not applicable.

\section{Competing interests}

The authors declare that they have no competing interests.

\section{Publisher's Note}

Springer Nature remains neutral with regard to jurisdictional claims in published maps and institutional affiliations.

\section{Author details}

'Duke University School of Nursing, 307 Trent Drive, Durham, NC, USA. ${ }^{2}$ Fudan University School of Nursing, Shanghai, China. ${ }^{3}$ Fudan University Center for Evidence-Based Nursing, a Joanna Briggs Institute Center of Excellence, Shanghai, China. ${ }^{4}$ New York University Rory Meyers College of Nursing, New York, NY, USA. ${ }^{5}$ Hartford Institute for Geriatric Nursing, New York University, New York, NY, USA. ${ }^{6}$ Geriatric Research, Education and Clinical Center (GRECC) of the Department of Veterans Affairs Medical Center, Durham, NC, USA.

Received: 1 June 2018 Accepted: 27 August 2018 Published online: 15 September 2018

\section{References}

1. $\quad$ Alzheimer's Disease International: World Alzheimer Report 2016. In.; 2016.

2. Prince $M$, Guerchet M, Prina M. The epidemiology and impact of dementia: current state and future trends. Geneva: World Health Organization; 2015.

3. Gates NJ, Sachdev PS, Singh MAF, Valenzuela M. Cognitive and memory training in adults at risk of dementia: a systematic review. BMC Geriatr. 2011;11(1):55.

4. The White House Office of the Press Secretary: FACT SHEET: the white house conference on Aging 2015. In.; 2015.

5. Petersen RC. Mild cognitive impairment as a diagnostic entity. J Intern Med. 2004;256(3):183-94.

6. Feldman $\mathrm{HH}$, Jacova C. Mild cognitive impairment. Am J Geriatr Psychiatry. 2005;13(8):645-55.

7. Jean L, Bergeron M-È, Thivierge S, Simard M. Cognitive intervention programs for individuals with mild cognitive impairment: systematic review of the literature. Am J Geriatr Psychiatry. 2010;18(4):281-96.

8. Zheng G, Xia R, Zhou W, Tao J, Chen L. Aerobic exercise ameliorates cognitive function in older adults with mild cognitive impairment: a systematic review and meta-analysis of randomised controlled trials. Br J Sports Med. 2016:50(23):1443.

9. Belleville S. Cognitive training for persons with mild cognitive impairment Int Psychogeriatr. 2008;20(1):57-66.

10. Reijnders J, van Heugten C, van Boxtel M. Cognitive interventions in healthy older adults and people with mild cognitive impairment: a systematic review. Ageing Res Rev. 2013;12(1):263-75.

11. Faucounau V, Wu Y-H, Boulay M, De Rotrou J, Rigaud A-S. Cognitive intervention programmes on patients affected by mild cognitive impairment: a promising intervention tool for $\mathrm{MCl}$ ? J Nutr Health Aging. 2010;14(1):31-5.

12. Hagovská M, Dzvoník O, Olekszyová Z. Comparison of two cognitive training programs with effects on functional activities and quality of life. Res Gerontol Nurs. 2017;10:172-80.

13. Man D, Chung J, Lee G: Evaluation of a virtual reality-based memory training programme for Hong Kong Chinese older adults with questionable dementia: a pilot study. 2012

14. Chandler MJ, Parks AC, Marsiske M, Rotblatt LJ, Smith GE. Everyday impact of cognitive interventions in mild cognitive impairment: a systematic review and meta-analysis. Neuropsychol Rev. 2016;26(3):225-51.

15. Coyle H, Traynor V, Solowij N. Computerized and virtual reality cognitive training for individuals at high risk of cognitive decline: systematic review of the literature. Am J Geriatr Psychiatr. 2015;23(4):335-59.

16. Hill NT, Mowszowski L, Naismith SL, Chadwick VL, Valenzuela M, Lampit A. Computerized cognitive training in older adults with mild cognitive impairment or dementia: a systematic review and meta-analysis. Am J Psychiatr. 2017;174(4):329-40.

17. Visser PJ, Kester A, Jolles J, Verhey F. Ten-year risk of dementia in subjects with mild cognitive impairment. Neurology. 2006;67(7):1201-7.

18. Moher D, Liberati A, Tetzlaff J, Altman DG, Group P. Preferred reporting items for systematic reviews and meta-analyses: the PRISMA statement. PLoS Med. 2009;6(7):e1000097.

19. Tufanaru C, Munn Z, Aromataris E, Campbell J, Hopp L: Chapter 3: Systematic reviews of effectiveness. In: Joanna Briggs Institute Reviewer's Manual The Joanna Briggs Institute, 2017. Edited by Aromataris E, Munn Z; 2017.

20. Nordmann AJ, Kasenda B, Briel M. Meta-analyses: what they can and cannot do. Swiss Med Wkly. 2012;142:W13518.

21. Greco T, Zangrillo A, Biondi-Zoccai G, Landoni G. Meta-analysis: pitfalls and hints. Heart, lung and vessels. 2013;5(4):219.

22. Barnes DE, Yaffe K, Belfor N, Jagust WJ, DeCarli C, Reed BR, et al. Computer-based cognitive training for mild cognitive impairment: results from a pilot randomized, controlled trial. Alzheimer Dis Assoc Disord. 2009;23:205-10

23. Rosen AC, Sugiura L, Kramer JH, Whitfield-Gabrieli S, Gabrieli JD. Cognitive training changes hippocampal function in mild cognitive impairment: a pilot study, vol. 2; 2011. p. 617-25.

24. RevMan. Copenhagen: the Nordic Cochrane Centre, the Cochrane collaboration. 5.3 ed; 2014.

25. Finn $\mathrm{M}, \mathrm{McD}$ Donald $\mathrm{S}$. Computerised cognitive training for older persons with mild cognitive impairment: a pilot study using a randomised controlled trial design. Brain Impairment. 2011;12(3):187-99.

26. Fiatarone Singh MA, Gates N, Saigal N, Wilson GC, Meiklejohn J, Brodaty H, et al. The study of mental and resistance training (SMART) study-resistance training and/or cognitive training in mild cognitive impairment: a randomized, double-blind, double-sham controlled trial. J Am Med Dir Assoc. 2014;15(12):873-80

27. Bahar-Fuchs A, Webb S, Bartsch L, Clare L, Rebok G, Cherbuin N, et al. Tailored and adaptive computerized cognitive training in older adults at risk for dementia: a randomized controlled trial. J Alzheimers Dis. 2017:60(3):889-911.

28. Hyer L, Scott C, Atkinson MM, Mullen CM, Lee A, Johnson A, et al. Cognitive training program to improve working memory in older adults with $\mathrm{MCl}$. Clinical Gerontologist: The Journal of Aging and Mental Health. 2016:39(5):410-27.

29. Cipriani G, Bianchetti A, Trabucchi M. Outcomes of a computer-based cognitive rehabilitation program on Alzheimer's disease patients compared with those on patients affected by mild cognitive impairment. Archives of Gerontology \& Geriatrics. 2006:43(3):327-35.

30. Han JW, Oh K, Yoo S, Kim E, Ahn KH, Son YJ, et al. Development of the ubiquitous spaced retrieval-based memory advancement and rehabilitation training program. Psychiatry investigation. 2014;11(1):52-8. 
31. Vermeij A, Claassen JA, Dautzenberg PL, Kessels RP. Transfer and maintenance effects of online working-memory training in normal ageing and mild cognitive impairment. Neuropsychol Rehabil. 2016;26(5-6):783-809.

32. Manera V, Petit PD, Derreumaux A, Orvieto I, Romagnoli M, Lyttle G, et al. 'Kitchen and cooking,' a serious game for mild cognitive impairment and Alzheimer's disease: a pilot study. Front Aging Neurosci. 2015;7:24.

33. Gonzalez-Palau F, Franco M, Bamidis P, Losada R, Parra E, Papageorgiou SG, et al. The effects of a computer-based cognitive and physical training program in a healthy and mildly cognitive impaired aging sample. Aging Ment Health. 2014;18(7):838-46.

34. Styliadis C, Kartsidis P, Paraskevopoulos E, loannides AA, Bamidis PD. Neuroplastic effects of combined computerized physical and cognitive training in elderly individuals at risk for dementia: an ELORETA controlled study on resting states. Neural plasticity. 2015;2015:172192.

35. Gooding AL, Choi J, Fiszdon JM, Wilkins K, Kirwin PD, van Dyck CH, et al. Comparing three methods of computerised cognitive training for older adults with subclinical cognitive decline. Neuropsychol Rehabil. 2016;26(5/6):810-21.

36. Talassi E, Guerreschi M, Feriani M, Fedi V, Bianchetti A, Trabucchi M. Effectiveness of a cognitive rehabilitation program in mild dementia (MD) and mild cognitive impairment (MCl): a case control study. Arch Gerontol Geriatr. 2007:44:391-9.

37. Klados MA, Styliadis C, Frantzidis CA, Paraskevopoulos E, Bamidis PD. Betaband functional connectivity is reorganized in mild cognitive impairment after combined computerized physical and cognitive training. Front Neurosci. 2016:10:55

38. Mansbach WE, Mace RA, Clark KM. The efficacy of a computer-assisted cognitive rehabilitation program for patients with mild cognitive deficits: a pilot study. Exp Aging Res. 2017;43:94-104.

39. Barban F, Annicchiarico R, Pantelopoulos S, Federici A, Perri R, Fadda $L$, et al. Protecting cognition from aging and Alzheimer's disease: a computerized cognitive training combined with reminiscence therapy. Int J Geriatr Psychiatry. 2016;31(4):340-8.

40. Rozzini L, Costardi D, Chilovi BV, Franzoni S, Trabucchi M, Padovani A. Efficacy of cognitive rehabilitation in patients with mild cognitive impairment treated with cholinesterase inhibitors. International Journal of Geriatric Psychiatry: A journal of the psychiatry of late life and allied sciences. 2007;22(4):356-60

41. Savulich G, Piercy T, Fox C, Suckling J, Rowe JB, O'Brien JT, et al. Cognitive training using a novel memory game on an iPad in patients with amnestic mild cognitive impairment (aMCl). Int J Neuropsychopharmacol. 2017:20:624-33

42. Herrera C, Chambon C, Michel BF, Paban V, Alescio-Lautier B. Positive effects of computer-based cognitive training in adults with mild cognitive impairment. Neuropsychologia. 2012;50(8):1871-81.

43. Gagnon LG, Belleville S. Training of attentional control in mild cognitive impairment with executive deficits: results from a double-blind randomised controlled study. Neuropsychol Rehabil. 2012;22(6):809-35.

44. Lin F, Heffner KL, Ren P, Tivarus ME, Brasch J, Chen DG, et al. Cognitive and neural effects of vision-based speed-of-processing training in older adults with amnestic mild cognitive impairment: a pilot study. J Am Geriatr Soc. 2016;64:1293-8.

45. Hughes TF, Flatt JD, Fu B, Butters MA, Chang CCH, Ganguli M. Interactive video gaming compared with health education in older adults with mild cognitive impairment: a feasibility study. Int I Geriatr Psychiatry. 2014;29(9):890-8.

46. Law LL, Barnett F, Yau MK, Gray MA. Effects of combined cognitive and exercise interventions on cognition in older adults with and without cognitive impairment: a systematic review. Ageing Res Rev. 2014;15:61-75.

47. Delbroek T, Vermeylen W, Spildooren J. The effect of cognitive-motor dual task training with the biorescue force platform on cognition, balance and dual task performance in institutionalized older adults: a randomized controlled trial. Journal of physical therapy science. 2017;29(7):1137-43.

Ready to submit your research? Choose BMC and benefit from:

- fast, convenient online submission

- thorough peer review by experienced researchers in your field

- rapid publication on acceptance

- support for research data, including large and complex data types

- gold Open Access which fosters wider collaboration and increased citations

- maximum visibility for your research: over $100 \mathrm{M}$ website views per year

At BMC, research is always in progress.

Learn more biomedcentral.com/submissions 